\title{
An advanced method for wind turbine wake modeling
}

\author{
Hüseyin Özdemir* and Edwin T.G. Bot ${ }^{\dagger}$ \\ Energy Research Center of the Netherlands (ECN), 1755 LE, Petten, The Netherlands
}

\begin{abstract}
In this study an advanced wake modeling for wind turbine wakes is presented. The wake model is based on the idea presented by Crespo where the Navier-Stokes equations are written in parabolic form with the assumption that the flow is dominant in the wake direction and there is no information traveling upstream. Various components of this model are presented in detail including near wake, root vortex and atmospheric boundary layer stability models. Furthermore the Active Wake Control concept is explained that modifies the pitch and yaw angles of upstream wind turbines in order to increase the overall power production and reduce the loading of the turbines. Finally a validation test case is presented where the results obtained from the wake model are compared to the lidar data obtained from an offshore wind farm.
\end{abstract}

\section{Introduction}

Wind turbines extract energy from the flow field which results in wakes that contain less energy and more turbulence than the undisturbed flow and this leads to less energy extraction and higher fatigue loads for the downstream turbines. In large wind farms, most turbines are located in the wake of one or more turbines. The overall loss of a wind farm is very much dependent on the conditions and the lay-out of the farm but it can be in the order of 5-20\%. Apart from the loss in energy production an additional wake effect is formed by the increase in turbulence intensity, which leads to higher fatigue loads. In this sense it becomes important to understand the details of the wake behavior to improve and/or optimize a wind farm layout. Within this study improvements are presented for the existing ECN wake model which constructs the fundamental basis of ECN's FarmFlow wind farm wake simulation tool. ${ }^{1-4}$

The ECN wake model is called WakeFarm ${ }^{1-4}$ which is based on the original UPMWAKE model proposed by Crespo et al. ${ }^{5,6}$ that simulates the wind turbine wakes by solving the steady parabolized Navier-Stokes equations in perturbation form in three-dimensions. The basic background flow is modeled by an atmospheric wind profile model based on Monin-Obukhov similarity theory. ${ }^{7}$ The similarity relations suggested by Businger $^{8}$ et al. are used. Because the axial-pressure gradient term is neglected in the parabolized NavierStokes equations, the perturbation variables are initialized by a near wake model.

Schepers ${ }^{1}$ pointed out the problem in the near wake and used an empirical velocity-deficit profile as a boundary condition for the far wake. This approach depends on a data-fit with experimental data and the physics of the flow are not modeled explicitly. Schepers and Van der Pijl ${ }^{3}$ proposed a model for the near wake based on the free-wake vortex method where the wind turbine is modeled by an actuator disc model and the wake is represented by discrete constant strength vortex rings. They obtained the solution with a panel method. A new near wake model is presented here based on a free wake-vortex method as well, where the radius of the wake and vorticity strength of discrete vortex rings are varied as suggested by Øye. ${ }^{9}$ The induced velocities are obtained by a semi-analytical solution of the Biot-Savart law.

The diabatic wind profiles for the surface layer of the atmospheric boundary layer have been investigated extensively. ${ }^{8,10}$ The atmospheric stability model based on Monin-Obukhov ${ }^{7}$ theory is only valid within the surface layer of the atmospheric boundary layer. Previous studies ${ }^{11-13}$ show that boundary layer height varies typically between $50-200 \mathrm{~m}$ under stable conditions and $500-1000 \mathrm{~m}$ under unstable conditions. A need for a model that extends to the entire boundary layer height is obvious considering the sizes of modern wind turbines. Blackadar ${ }^{14}$ and Lettau ${ }^{15}$ studied a wind shear model covering the entire boundary layer

\footnotetext{
${ }^{*}$ Researcher, Wind Energy Unit, Westerduinweg 3, h.ozdemir@ecn.nl. Member AIAA

${ }^{\dagger}$ Researcher, Wind Energy Unit, Westerduinweg 3, bot@ecn.nl. Member AIAA
} 
height under neutral conditions. Gryning et al. ${ }^{16}$ extended this model to cover all stability conditions of the atmosphere based on measurements extending into the mixing layer region where the surface layer scaling is connected with the geostrophic drag law. More recently similar work is done by Peña et al. ${ }^{17}$ Sathe $e t$ al. ${ }^{18}$ showed that the loads are predicted smaller with the model proposed by Gryning when compared to models based only on surface layer wind profiles.

Within this study the ECN wake model is extended further based on the model proposed by Gryning et $a l .{ }^{16}$ The numerical solution obtained by the ECN wake model using Gryning model is compared with the solution obtained by surface layer model and with the available data obtained by measurements on ECN's Wind turbine Test field Wieringermeer (EWTW).

Active Wake Control (AWC) is an approach of operating wind farms with the aim at improving the overall wind farm performance in terms of power production at below rated wind conditions. It consists of a pitch-based approach (also known as Heat \& Flux), and a yaw-based approach (called Controlling Wind). In this study the AWC concept is explained in details and furthermore a method to apply this method in a quasi-dynamic way is briefly discussed.

The outline of this paper is as follows: First of all the governing equations of the ECN wake farm model are presented. Then the new near wake modeling is discussed and the results compared with the original near wake modeling and EWTW data as well as the results obtained for various near wake implementation cases are shown. The details of the atmospheric stability model are given and the comparison with the solution obtained for the original surface layer model and with the available data obtained by EWTW measurements are presented. Details of AWC concept and quasi-dynamic implementation is given. Some recent results of a validation test case is presented and finally the conclusions are summarized.

\section{Governing Equations}

In the wake model originally proposed by Crespo et al. ${ }^{5}$ the wind turbine is supposed to be immersed in a nonuniform basic flow corresponding to the surface layer of the atmospheric boundary layer. This surface layer which is modeled by the standard surface-layer scaling based on the Monin-Obukhov theory, is assumed to be perturbed by the wind turbine. The equations describing the perturbed flow are obtained by introducing the following perturbation variables into the Navier-Stokes equations together with the conservation of mass and energy equations and two equations for turbulent kinetic energy and the dissipation rate of the turbulent kinetic energy:

$$
u=u^{\prime}+u_{0}, \quad v=v^{\prime}, \quad w=w^{\prime}, \quad p=p^{\prime}+p_{0}, \quad \theta=\theta^{\prime}+\theta_{0}, \quad k=k^{\prime}+k_{0}, \quad \epsilon=\epsilon^{\prime}+\epsilon_{0},
$$

with $u, v$, and $w$ are three components of the velocity vector, $p$ is the pressure, $\theta$ is the potential temperature, $k$ is the turbulent kinetic energy and $\epsilon$ is the turbulent dissipation rate. The subscript " 0 " refers to the undisturbed flow and the superscript ()$^{\prime}$ refers to a perturbation variable. This undisturbed flow does not vary in $x$ - and $y$-directions, but does vary in $z$ direction.

For the wake flow behind a wind turbine the following assumptions are made: the streamwise pressure gradient is neglected in the far wake behind the wind turbine while the near wake is modeled by the freevortex wake model as part of basic background flow. The streamwise diffusion is neglected leading to

$\frac{\partial \tau_{i j}}{\partial x}=0$ and $\nu_{t} \frac{\partial u_{j}}{\partial x}=0$. The undisturbed flow in $y$ - and $z$ - directions is assumed to be zero meaning that the information is only traveling downstream. Furthermore the turbulent stresses can be modeled by Boussinesq's eddy viscosity approximation:

$$
\tau_{i j}=\rho \nu_{t}\left[\frac{u_{i}}{x_{j}}+\frac{u_{j}}{x_{i}}\right]-\frac{2}{3} \rho k \delta_{i j} .
$$

Under these assumption the Navier Stokes equations that describe the flow can be written in parabolized form where the elliptic terms are not present anymore. When the Navier-Stokes equations for the undisturbed flow are subtracted from those equations for the disturbed flow, the following relations can be derived. Please note that the superscript ( $)^{\prime}$ is dropped for convenience.

Continuity equation:

$$
\frac{\partial u}{\partial x}+\frac{\partial v}{\partial y}+\frac{\partial w}{\partial z}=0
$$


Momentum equations ( $x, y$ and $z$ directions):

$$
\begin{gathered}
\left(u_{0}+u\right) \frac{\partial u}{\partial x}+v \frac{\partial u}{\partial y}+w \frac{\partial\left(u_{0}+u\right)}{\partial z}=\left(\nu_{t}+\nu_{t 0}\right) \frac{\partial^{2} u}{\partial y^{2}}+\frac{\partial \nu_{t}}{\partial y} \frac{\partial u}{\partial y} \\
+\left(\nu_{t}+\nu_{t 0}\right) \frac{\partial^{2} u}{\partial z^{2}}+\left(\frac{\partial \nu_{t}}{\partial z}+\frac{\partial \nu_{t 0}}{\partial z}\right) \frac{\partial u}{\partial z}+\frac{\partial \nu_{t}}{\partial z} \frac{\partial u_{0}}{\partial z}+\nu_{t} \frac{\partial^{2} u_{0}}{\partial z^{2}} \\
\left(u_{0}+u\right) \frac{\partial v}{\partial x}+v \frac{\partial v}{\partial y}+w \frac{\partial v}{\partial z}=-\frac{2}{3} \frac{\partial k}{\partial y}-\frac{1}{\rho} \frac{\partial p}{\partial y}+2\left(\nu_{t}+\nu_{t 0}\right) \frac{\partial^{2} v}{\partial y^{2}}+2 \frac{\partial \nu_{t}}{\partial y} \frac{\partial v}{\partial y} \\
+\left(\nu_{t}+\nu_{t 0}\right) \frac{\partial^{2} v}{\partial z^{2}}+\left(\frac{\partial \nu_{t}}{\partial z}+\frac{\partial \nu_{t 0}}{\partial z}\right) \frac{\partial v}{\partial z}+\left(\frac{\partial \nu_{t}}{\partial z}+\frac{\partial \nu_{t 0}}{\partial z}\right) \frac{\partial w}{\partial y} \\
\left(u_{0}+u\right) \frac{\partial w}{\partial x}+v \frac{\partial w}{\partial y}+w \frac{\partial w}{\partial z}=-\frac{1}{\rho} \frac{\partial p}{\partial z}+\left(\nu_{t}+\nu_{t 0}\right) \frac{\partial^{2} w}{\partial y^{2}}+\frac{\partial \nu_{t}}{\partial y} \frac{\partial w}{\partial y} \\
+2\left(\nu_{t}+\nu_{t 0}\right) \frac{\partial^{2} w}{\partial z^{2}}+2\left(\frac{\partial \nu_{t}}{\partial z}+\frac{\partial \nu_{t 0}}{\partial z}\right) \frac{\partial w}{\partial z}+\frac{\partial \nu_{t}}{\partial y} \frac{\partial v}{\partial z}-\frac{2}{3} \frac{\partial\left(k+k_{0}\right)}{\partial z}+\beta g \theta
\end{gathered}
$$

with $\beta$ is the expansion coefficient.

Energy equation:

$$
\begin{aligned}
\left(u_{0}+u\right) \frac{\partial \theta}{\partial x} & +v \frac{\partial \theta}{\partial y}+\frac{\partial\left(\theta_{0}+\theta\right)}{\partial z}=\left(\nu_{\theta}+\nu_{\theta 0}\right) \frac{\partial^{2} \theta}{\partial y^{2}}+w \frac{\partial \nu_{\theta}}{\partial y} \frac{\partial \theta}{\partial y} \\
& +\left(\nu_{\theta}+\nu_{\theta 0}\right) \frac{\partial^{2} \theta}{\partial z^{2}}+\left(\frac{\partial \nu_{\theta}}{\partial z}+\frac{d \nu_{\theta 0}}{d z}\right) \frac{\partial \theta}{\partial z}+\frac{\partial \nu_{\theta}}{\partial z} \frac{\partial \theta_{0}}{\partial z}+\nu_{\theta} \frac{\partial^{2} \theta_{0}}{\partial z^{2}}
\end{aligned}
$$

Turbulent kinetic energy:

$$
\begin{aligned}
& \left(u_{0}+u\right) \frac{\partial k}{\partial x}+v \frac{\partial k}{\partial y}+w \frac{\partial\left(k_{0}+k\right)}{\partial z}=\left(\nu_{k}+\nu_{k 0}\right) \frac{\partial^{2} k}{\partial y^{2}}+\frac{\partial \nu_{k}}{\partial y} \frac{\partial k}{\partial y}+\left(\nu_{k}+\nu_{k 0}\right) \frac{\partial^{2} k}{\partial z^{2}} \\
& \quad+\left(\frac{\partial \nu_{k}}{\partial z}+\frac{d \nu_{k 0}}{d z}\right) \frac{\partial k}{\partial z}+\frac{\partial \nu_{k}}{\partial z} \frac{\partial k_{0}}{\partial z}+\nu_{k} \frac{\partial^{2} k_{0}}{\partial z^{2}}+\left(\nu_{t}+\nu_{t 0}\right)\left[\left(\frac{\partial u}{\partial y}\right)^{2}+\left(\frac{\partial u}{\partial z}\right)^{2}+2 \frac{\partial u}{\partial z} \frac{\partial u_{0}}{\partial z}\right] \\
& \quad+\nu_{t}\left(\frac{d u 0}{d z}\right)^{2}-\beta g \nu_{\theta}\left(\frac{\partial \theta}{\partial z}+\frac{\partial \theta_{0}}{\partial z}\right)-\beta g \nu_{\theta 0} \frac{\partial \theta}{\partial z}-\epsilon .
\end{aligned}
$$

Dissipation rate of the turbulent kinetic energy:

$$
\begin{aligned}
& \left(u_{0}+u\right) \frac{\partial \epsilon}{\partial x}+v \frac{\partial \epsilon}{\partial y}+w \frac{\partial\left(\epsilon_{0}+\epsilon\right)}{\partial z}= \\
& \left(\nu_{\epsilon}+\nu_{\epsilon 0}\right) \frac{\partial^{2} \epsilon}{\partial y^{2}}+\frac{\partial \nu_{\epsilon}}{\partial y} \frac{\partial \epsilon}{\partial y}+\left(\nu_{\epsilon}+\nu_{\epsilon 0}\right) \frac{\partial^{2} \epsilon}{\partial z^{2}}+\left(\frac{\partial \nu_{\epsilon}}{\partial z}+\frac{d \nu_{\epsilon 0}}{d z}\right) \frac{\partial \epsilon}{\partial z}+\frac{\partial \nu_{\epsilon}}{\partial z} \frac{\partial \epsilon_{0}}{\partial z}+\nu_{\epsilon} \frac{\partial^{2} \epsilon_{0}}{\partial z^{2}} \\
& +C_{\epsilon_{1}} \frac{\epsilon_{0}+\epsilon}{k_{0}+k}\left(\nu_{t}+\nu_{t 0}\right)\left[\left(\frac{\partial u}{\partial y}\right)^{2}+\left(\frac{\partial u}{\partial z}\right)^{2}+\left(\frac{\partial u_{0}}{\partial z}\right)^{2}+2 \frac{\partial u}{\partial z} \frac{\partial u_{0}}{\partial z}-\left(1-C_{\epsilon_{3}}\right) \beta g \frac{1}{\sigma_{\theta}} \frac{\partial\left(\theta+\theta_{0}\right)}{\partial z}\right] \\
& -C_{\epsilon_{1}} \frac{\epsilon_{0}}{k_{0}} \nu_{t 0}\left[\left(\frac{\partial u_{0}}{\partial z}\right)^{2}-\left(1-C_{\epsilon_{3}}\right) \beta g \frac{1}{\sigma_{\theta}} \frac{\partial \theta_{0}}{\partial z}\right]-C_{\epsilon_{2}} \frac{\left(\epsilon+\epsilon_{0}\right)^{2}}{k+k_{0}}+C_{\epsilon_{2}} \frac{\epsilon_{0}^{2}}{k_{0}},
\end{aligned}
$$

with,

$$
\nu_{t}=C_{\mu} \frac{\left(k+k_{0}\right)^{2}}{\epsilon+\epsilon_{0}}-\nu_{t 0}, \quad \nu_{\theta}=\frac{\nu_{t}}{\sigma_{\theta}}, \quad \nu_{k}=\frac{\nu_{t}}{\sigma_{k}}, \quad \nu_{\epsilon}=\frac{\nu_{t}}{\sigma_{\epsilon}} .
$$

where $C_{\mu}, C_{\epsilon_{1}}, C_{\epsilon_{2}}, C_{\epsilon_{3}}, \sigma_{\theta}, \sigma_{k}$ and $\sigma_{\epsilon}$ are the closure coefficients of the system. The system of equations given between equation (3) and (9) form a steady, three-dimensional parabolic set of equations. These equations are discretized with central differences and integrated employing the semi-implicit ADI scheme. The pressure is evaluated using the SIMPLE method. ${ }^{19}$ 
Dirichlet and Neumann boundary conditions are applied at boundaries of the domain. ${ }^{6}$ The numerical values are determined from the undisturbed flow in front of the wind turbine.

The solution domain size is chosen to be at least ten times the wind turbine diameter, $\mathrm{D}$, along $x-$ direction (i.e., downstream wake direction) and six times the wind turbine diameter in $y$ - and $z$-directions. A grid stretching is applied along the $x$-direction within the near-wake region. For a typical simulation of a single turbine-single wake combination around $1 \cdot 10^{6}$ grid points are used.

\section{Wake Models}

\section{III.A. Near Wake Model}

In the original ECN wake model the near wake is modeled by a free-wake vortex model where the vorticity strength of vortex rings are kept constant while the wake radius is varied. The solution is obtained by a panel method. In the current study the near-wake model is improved further as suggested by Øye. ${ }^{9}$ The wind turbine rotor is modeled by an actuator disc model and the wake is represented by a thin vortex sheet which is described by vortex rings of variable radius and variable vorticity strength leading to a variable transport velocity at infinity. Flow is assumed to be axisymmetric. Induced velocity profiles are obtained analytically using Biot-Savart law, where the elliptic integrals are evaluated numerically. The radii along the wake are calculated by making use of the continuity equation and the solution is iterated until convergence is reached between the local radius and the vorticity.

In figure (1) a comparison of various calculation methods that have been studied and the original panel method are presented. The wake radius is plotted against the distance from the turbine in downstream direction for constant vortex strength $(\Gamma)$ and variable vortex strength cases for uniform and stretched grid distributions. All results are obtained for an axial induction factor of 0.28 . The left-hand side of the figure is a close-up of the near-wake region while on the right-hand side the full domain is presented. In figure (2)
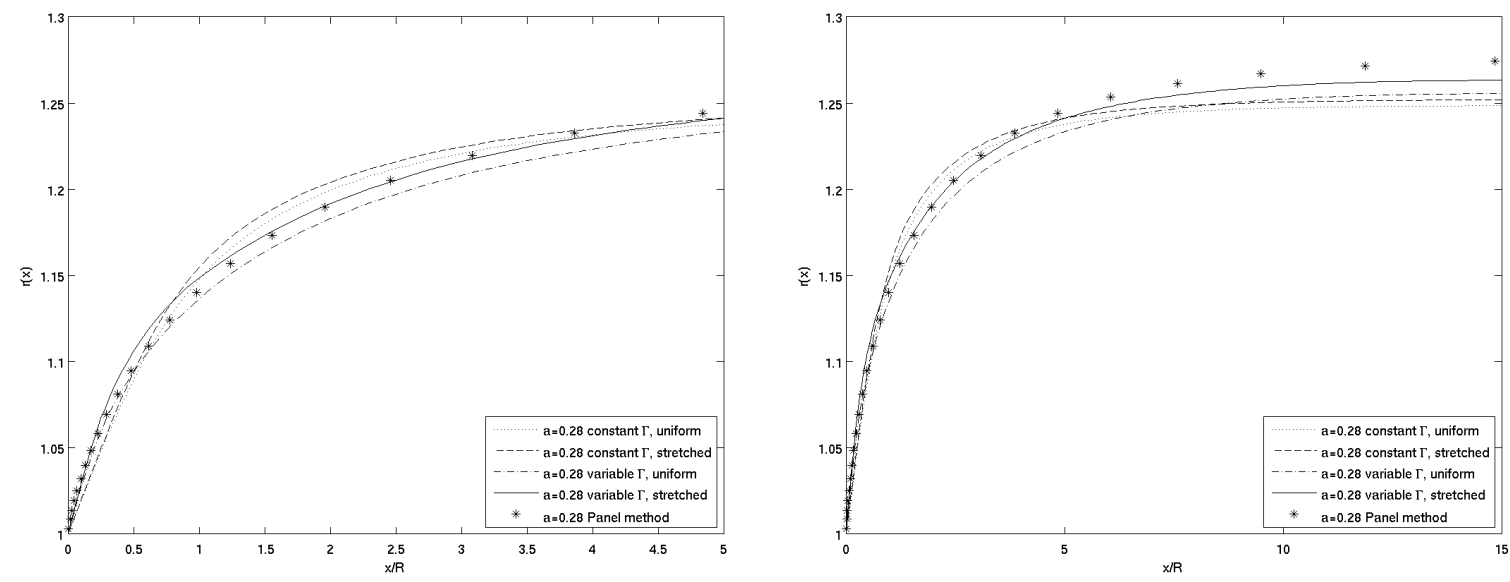

Figure 1. Comparison of the wake radius as a function of distance from the turbine up to $5 R$ (left) and $15 R$ (right) with $a=0.28$, as simulated by the constant vorticity vortex ring model, the variable vorticity vortex ring model and the panel method. The new near wake models are simulated on uniform and stretched grids.

the induced velocity just behind the rotor is shown for the panel method and the variable vorticity vortex ring method for an axial induction factor of 0.28 . It is clear that the models are comparable, but that inside the wake the induced velocity in downstream direction is higher for the variable vortex ring method in an absolute sense. According to actuator disc momentum theory the induced velocity just behind the rotor is $-a U_{\infty}$, so the variable vorticity vortex ring model agrees better with the theory. The results of both original and new wake models are compared with the experimental data obtained from the measurements at ECN Wind turbine Test field Wieringermeer, EWTW. The test site consists of five state of the art turbines in a row, a scaled wind farm with 10 wind turbines of 7.5 meter hight and 7.6 meter in diameter and 7 prototype spots for certification of industrial turbines. The five turbines, with a hub height and diameter of 80 meters, 


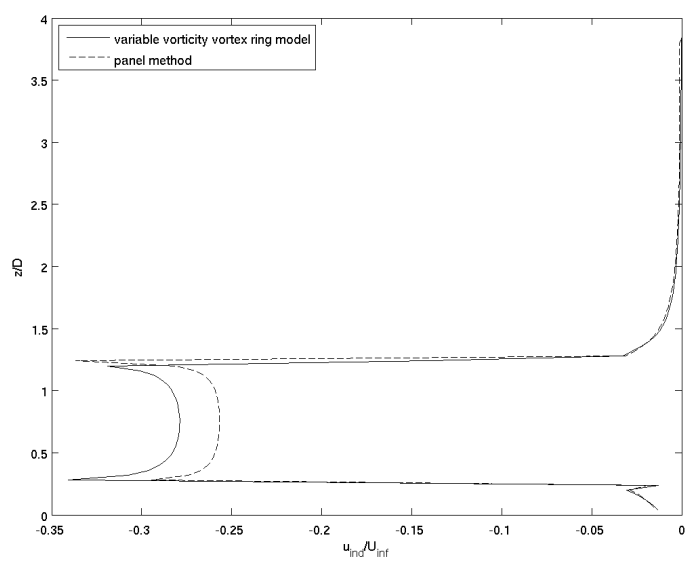

Figure 2. Comparison of velocity and force profiles just behind the rotor plane at the symmetry plane, as simulated by the variable vorticity vortex ring model (the new near wake model) and the panel method (the old near wake model).

are situated in a row and a meteorological measurement mast is located in the proximity of $\mathrm{T} 5$ and $\mathrm{T} 6$, as can be seen on the left-hand side of figure (3). ${ }^{20}$ Since there is only one measured point in the field, multiple measurements are combined to get the horizontal wake profile behind a turbine on a certain distance. The right-hand side of figure (3) shows the distance $S$ between the mast and the wind turbines: 2.5D and 3.5D. When the wind direction is 31 degrees or 315 degrees the velocity at the wake center is measured. When the wind comes from a different direction, the distance $y$ to the wake center is calculated. The results of the
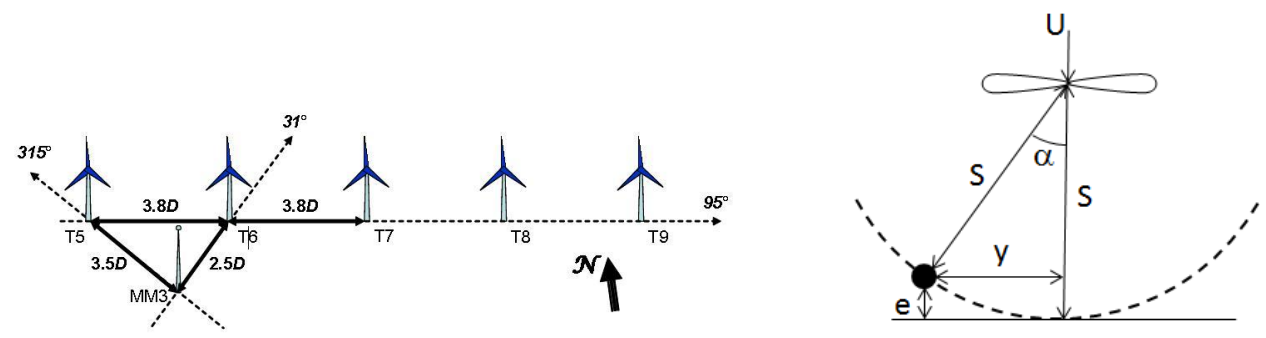

Figure 3. Location of wind turbines and meteorological measurement mast at EWTW (left). When the turbine yaws the meteorological mast moves along the dashed line with respect to the rotor (right).

numerical simulation obtained by the ECN wake model WakeFarm using the original (panel method) and new near wake models are compared with the EWTW data and shown in figure (4). WakeFarm performs best at $2.5 \mathrm{D}$, but for $3.5 \mathrm{D}$ the results deviate more from the experimental measurements. For the details of the experimental work one is referred to reference. ${ }^{20}$

\section{III.B. Atmospheric Boundary Layer Stability Model}

In the original UPMWAKE model of Crespo et al. ${ }^{5}$ a diabatic wind profile based on Monin-Obukhov ${ }^{7}$ similarity theory and Businger et al. ${ }^{8}$ formulation is used given as follows:

$$
u=\frac{u_{* 0}}{\kappa}\left[\ln \left(\frac{z}{z_{0}}\right)-\psi_{m}(z / L)\right]
$$

where $u_{* 0}$ is the friction velocity near the ground, $\mathrm{z}$ is the height, $z_{0}$ is the aerodynamic roughness length, $\kappa$ is the von Kármán constant, $L$ is the Monin-Obukhov length and $\psi_{m}$ is a universal stability function which is defined empirically. In the original ECN wake model the above wind profile is adopted whith the Bussinger et al. ${ }^{8}$ form for stability function $\psi_{m}$ where further details are given by Panofsky and Dutton. ${ }^{21}$ 

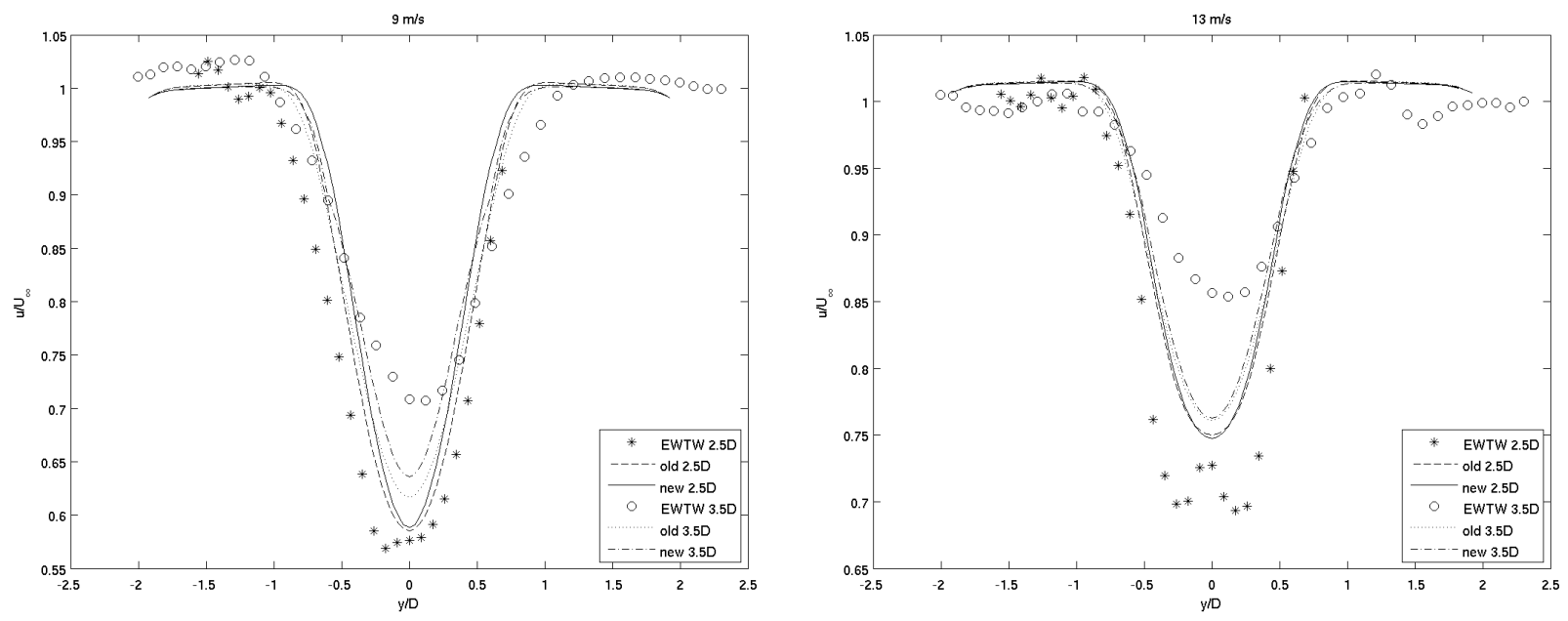

Figure 4. Comparison of EWTW experimental data with velocity profiles in wake direction, as simulated by ECN wake model using the original and the new near wake model, respectively.

The Monin-Obukhov length, $L$, is given by the following expression:

$$
L=\frac{u_{* 0}^{3} T}{\kappa g \overline{\omega^{\prime} \theta_{0}^{\prime}}}
$$

where, $T$ is the absolute temperature, $\theta_{0}$ is the potential temperature and $\overline{\omega^{\prime} \theta_{0}^{\prime}}$ is the virtual kinematic heat flux.

The diabatic wind profile models using surface layer scaling of Monin-Obukhov ${ }^{7}$ are valid only within the surface layer of the atmosphere. In the current study the extended wind profile model proposed by Gryning et $a .^{16}$ is used which is valid for the entire boundary layer. The model is given as follows:

For neutral conditions:

$$
u=\frac{u_{* 0}}{\kappa}\left[\ln \left(\frac{z}{z_{0}}\right)+\frac{z}{L_{M B L}}-\frac{z}{z_{i}}\left(\frac{z}{2 L_{M B L}}\right)\right]
$$

for unstable conditions:

$$
u=\frac{u_{* 0}}{\kappa}\left[\ln \left(\frac{z}{z_{0}}\right)-\psi_{m}(z / L)+\frac{z}{L_{M B L}}-\frac{z}{z_{i}}\left(\frac{z}{2 L_{M B L}}\right)\right]
$$

for stable conditions:

$$
u=\frac{u_{* 0}}{\kappa}\left[\ln \left(\frac{z}{z_{0}}\right)-\psi_{m}(z / L)\left(1-\frac{z}{2 z_{i}}\right)+\frac{z}{L_{M B L}}-\frac{z}{z_{i}}\left(\frac{z}{2 L_{M B L}}\right)\right],
$$

with $z_{i}$ is the height of the atmospheric boundary layer and defined as follows for the neutral conditions: ${ }^{22}$

$$
z_{i}=c \frac{u_{* 0}}{|f|}
$$

with $c$ is a constant and $f$ is the Coriolis parameter. The value of $c$ is adopted from the works of Sathe $e t$ $a l .{ }^{18}$ and Peña et al. ${ }^{23,24}$ as 0.15 for neutral conditions, 0.14 for stable conditions and 0.13 for very stable conditions.

Stability function, $\psi_{m}$, is used from Businger et al. ${ }^{8}$ for stable conditions and from Grachev et al. ${ }^{10}$ for unstable conditions. An empirical fit suggested by Gryning et al. ${ }^{16}$ for the scaling parameter, $L_{M B L}$, is in the following form:

$$
\frac{u_{* 0}}{f L_{M B L}}=\left(-2 \ln \left(\frac{u_{* 0}}{f z_{0}}\right)+55\right) e^{\left(-\frac{\left(u_{* 0} / f L\right)^{2}}{400}\right)} .
$$


In figure (5) the influence of various models for an atmospheric boundary layer velocity profile is presented using the parameters listed in table (1). It can also be concluded from these figures that the choice for the atmospheric boundary layer stability model is most important for (very) stable conditions.

\begin{tabular}{l|lll} 
& Unstable & Neutral & Stable \\
\hline $\mathbf{L}$ & $-128 \mathrm{~m}$ & $321 \mathrm{~m}$ & $41 \mathrm{~m}$ \\
$\mathbf{z}_{\mathbf{i}}$ & $117 \mathrm{~m}$ & $205 \mathrm{~m}$ & $49 \mathrm{~m}$ \\
$\mathbf{L}_{\mathbf{M B L}}$ & $283 \mathrm{~m}$ & $866 \mathrm{~m}$ & $69 \mathrm{~m}$ \\
\multicolumn{3}{c}{ Table 1. Parameters used for figure (5) }
\end{tabular}

Sathe $e$ al. ${ }^{18}$ showed the importance of using a diabatic wind profile for the entire boundary layer. They argued that using a surface layer model predicts the blade and rotor loads along the wake direction much larger compared to a model for the entire boundary layer particularly for stable conditions, since in the surface-layer wind profile model under stable conditions, the wind profile length scale

increases infinitely, leading to large wind gradients, while a wind profile for the entire boundary layer ${ }^{16}$ limits the growth of this length scale using the boundary layer height, leading to smaller wind shear.

In figure (6) a comparison of numerical simulation using Gryning et al. ${ }^{16}$ model valid for the entire boundary layer for stable, neutral and unstable conditions with the surface layer model and the available data from EWTW measurements for $U_{\infty}=8 \mathrm{~m} / \mathrm{s}$ (left) and $U_{\infty}=10 \mathrm{~m} / \mathrm{s}$ (right) is given. The EWTW data is evaluated as described in the previous section. The induced velocity is predicted lower compared to the surface layer model which is in agreement with Sathe et al. ${ }^{18}$ It should be noted here that a single EWTW data set is used for the entire stability region and the simulation results for each stability region are compared with this single data.

There is a necessity of determination of the experimental data for various stability conditions. The available EWTW data do not contain any information to categorize the stability of the atmosphere of the measurements at the moment. In the literature several rough estimates are given about the estimation of the stability condition of the atmosphere depending on the time of the day. ${ }^{22}$ Based on these rough estimates the data measured between sunrise and sunset is assumed to be unstable and between sunset and sunrise is assumed to be stable. The comparison of the numerical simulation using Gryning et al. ${ }^{16}$ model with the surface layer model and the data from EWTW measurements for stable and unstable conditions for $U_{\infty}=8 \mathrm{~m} / \mathrm{s}$ is shown in figure

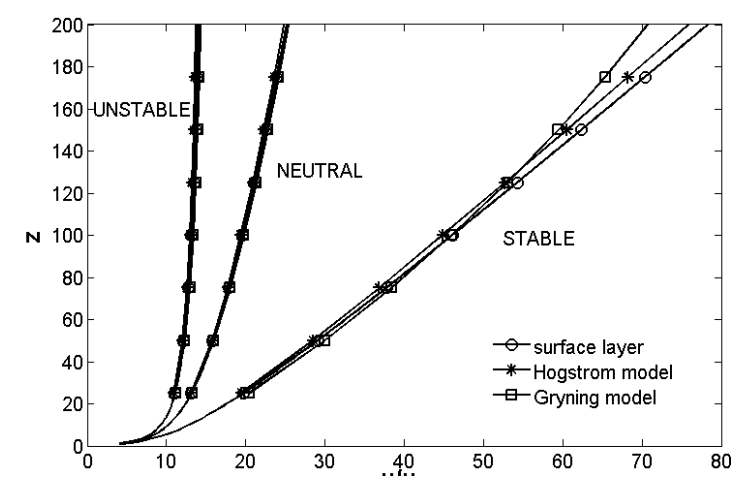

Figure 5. Influence of the atmospheric boundary-layer model on the velocity profile for unstable, neutral and stable conditions. (7) at $2.5 D$ and $3.5 D$ downstream of the turbine. Although the comparison is not conclusive because of the reliability of the data it is shown here for the demonstration purposes.

\section{Active Wake Control Concept}

Active Wake Control (AWC) is an approach of operating wind farms in such a way as to maximize the overall wind farm power production. It consists of two concepts: pitch-based AWC (called Heat \& Flux), and yaw-based AWC (called Controlling Wind).

\section{IV.A. Heat \& Flux}

The Heat \& Flux concept ${ }^{20,25}$ is a wind farm operating strategy for improving the power output and reducing the loads of the wind farm as a whole. The term heat reflects the energy dissipation (as heat) when two flows of air with different velocities mix, i.e. the stream tube around the rotor and the outer flow. Flux refers to the flow of air through the farm. The general idea behind the concept is to reduce the heat losses and the flux deficit of an aligned row of wind turbines by lowering the induction of upstream turbines. Reducing 

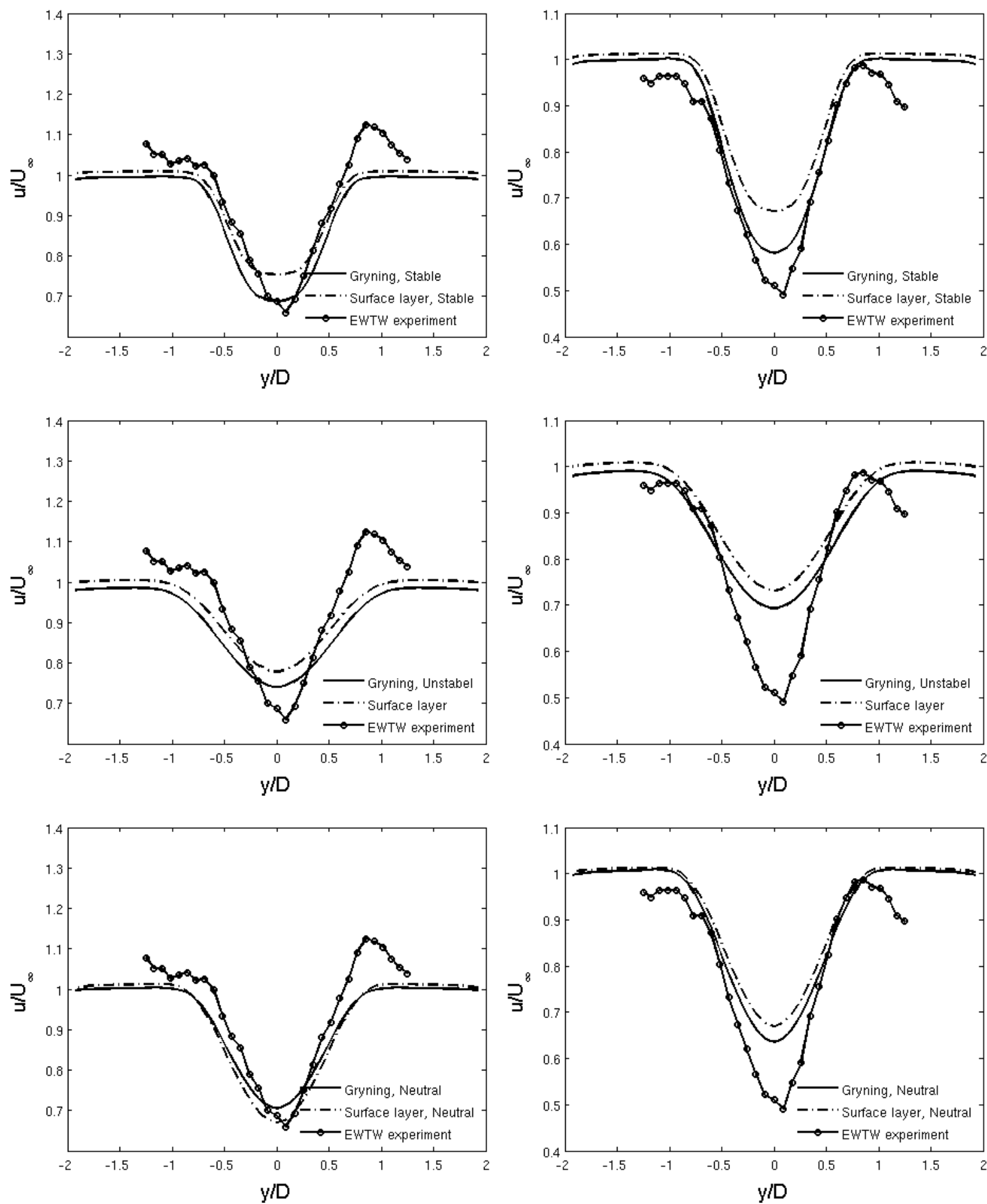

Figure 6. Comparison of numerical simulation using Gryning et al. ${ }^{16}$ model for stable, neutral and unstable conditions with the surface layer model and the available data from EWTW measurements for $U_{\infty}=8 \mathrm{~m} / \mathrm{s}($ left $)$ and $U_{\infty}=10 \mathrm{~m} / \mathrm{s}$ (right).

the induction can be achieved by changing (either one or both) the turbine's blade pitch angle $\theta_{b}$ and tip speed ratio $\lambda$. While the upstream turbine(s) will produce less, the downstream turbines make up for this, resulting in a net increase of power output. Also the loads reduce and are more evenly distributed over the turbines.

In a previous study ${ }^{25}$ the concept is validated in the boundary layer wind tunnel at TNO Apeldoorn. 

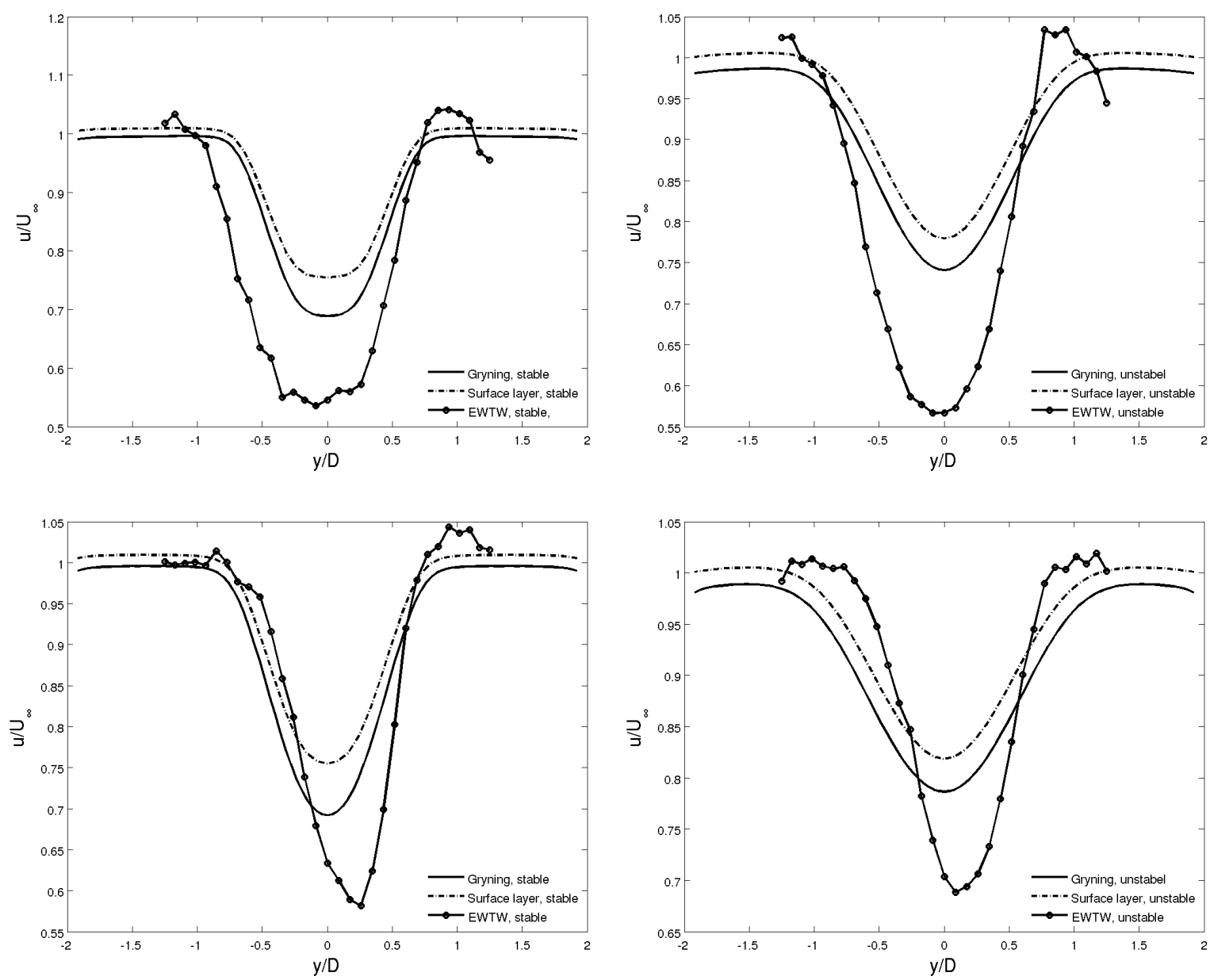

Figure 7. Comparison of numerical simulation using Gryning et al. ${ }^{16}$ model with the surface layer model and the data from EWTW measurements for stable and unstable conditions for $U_{\infty}=8 \mathrm{~m} / \mathrm{s}$, at $2.5 D$ (left) and $3.5 D$ (right).

Despite the scaling issues (rotor diameter of $25 \mathrm{~cm}$ ), this test under controlled conditions clearly demonstrated the effect. The measured increase in power output between the Heat \& Flux and reference row was about $4 \%$ (although this could be partly due to scaling effects). Also, it is worth to mention that the scatter in the data was large. The next step was a full scale test on EWTW. ${ }^{20}$ Although no optimal settings could be used, this test still showed power increase and load reduction for the aligned Heat \& Flux case. Unfortunately, these measurements were taken with difference in turbulence intensities, which should be accounted for.

Recently, a study by Küçükşahin ${ }^{26}$ quantified the effect of spacing and turbulence intensity on the performance of Heat \& Flux strategy. Increased spacing of the turbines reduces the power increase gained from Heat \& Flux. The simulation results on the EWTW indicate a power increase of about $3.5 \%$ at best for the Heat \& Flux scenarios compared to the base case. Increasing the spacing between the turbines to $8 D$, the benefit from Heat \& Flux reduces to $0.2 \%$ increase of energy yield. The turbulence has a sweet spot at about $9 \%$ for the test farm EWTW.

It should be noted that the optimal Heat \& Flux pitch angle depends on the wind direction, since this is the parameter that determines the spatial configuration of the wind farm. As a result, Heat \& Flux has the highest effect when the wind direction is such that a large number of wind turbines are in the wake of upstream wind turbine at a distance of less than several rotor diameters. On the other hand, when this wind direction condition is not satisfied, Heat \& Flux has little to no effect on the overall wind farm power production and loads.

If there would be no turbulence in the wakes (and therefore no mixing with the surrounding undisturbed air flow), the optimum induction (and thus blade pitch angle) could be estimated with the relation between thrust and induction as shown in. ${ }^{27}$ Of course, this is not the case in reality, and therefore in this study an 
accurate wake modelling tool, FarmFlow, is used to determine the optimum pitch angle setting.

For constant lambda operation, the farm effects (and therefore the optimal heat and flux pitch angle setting) are not wind speed dependent. Figure 8 shows the tip speed ratio as function of the wind speed for a $6 \mathrm{MW}$ wind turbine, which indicates this assumption is valid up to about $9 \mathrm{~m} / \mathrm{s}$. The optimal heat and flux pitch angle can thus be determined at a single wind speed below rated (and used for all below rated wind speeds). The relation between tip speed ratio and wind speed has been determined using the given rotor

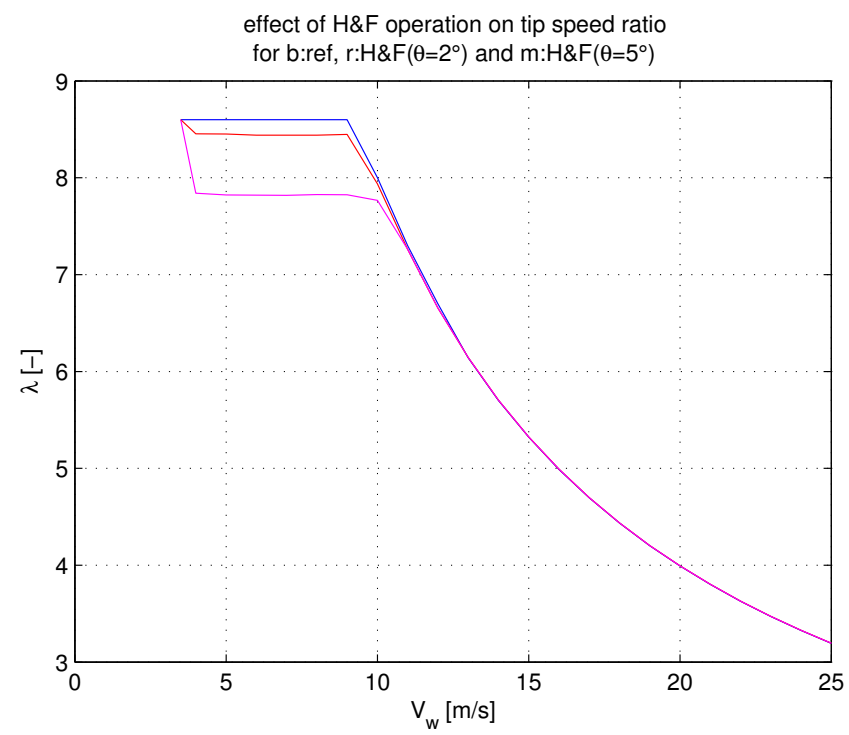

Figure 8. Tip speed ratio as function of wind speed for three different below-rated operation modes: optimum pitch angle (blue line), pitch angle offset of 2 degrees (red) and 4 degrees (magenda)

characteristics and the power curve (PV curve), estimated losses and assuming that the generator torque control remains unmodified under Heat \& Flux operation, i.e. the nominal torque-speed curve (QN-curve), exemplified in Figure 9, remains the same when the pitch angle is increased by Heat \& Flux. To calculate the tip-speed ratio for a given pair of wind speed and pitch angle, the rotor speed is first computed. To do this, the aerodynamic torque is calculated as function of the rotor speed using the rotor torque coefficient $C_{Q}$ for the considered wind speed and pitch angle. The rotor speed is then obtained by determining the crossing point of the curve aerodynamic torque - rotor speed and QN curve, from which the tip-speed ratio follows.

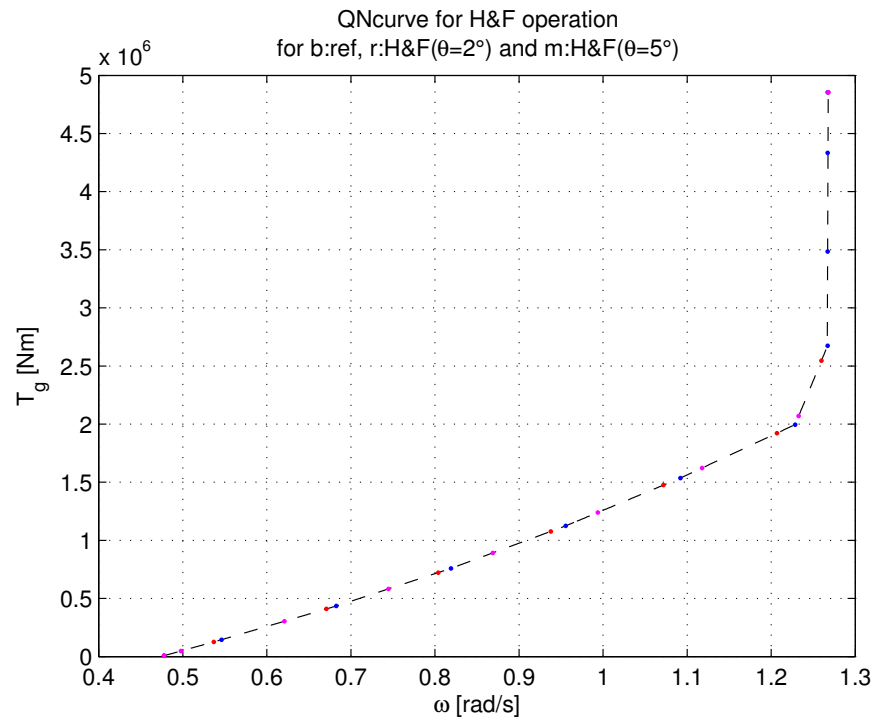

Figure 9. Equal generator torque control (QN-curve) assumed for normal and Heat \& Flux operation 


\section{IV.B. Controlling Wind}

The Controlling Wind concept ${ }^{28}$ is an alternative approach to control the wakes in a wind farm by applying yaw misalignment, i.e. yawing the rotors of the upstream wind turbines away from the wind. As a result of that, two things happen: the power production of the yawed wind turbines decreases because the effective rotor area affected by the incoming wind flow becomes smaller, and the wakes behind these turbines is redirected aside from the downstream wind turbines (see Figure 10). Due to the fact that the wake is being diverted, the downstream turbines can get (a larger portion of) the undisturbed wind field instead of the wake of the upstream wind turbine, which increases their power production substantially.
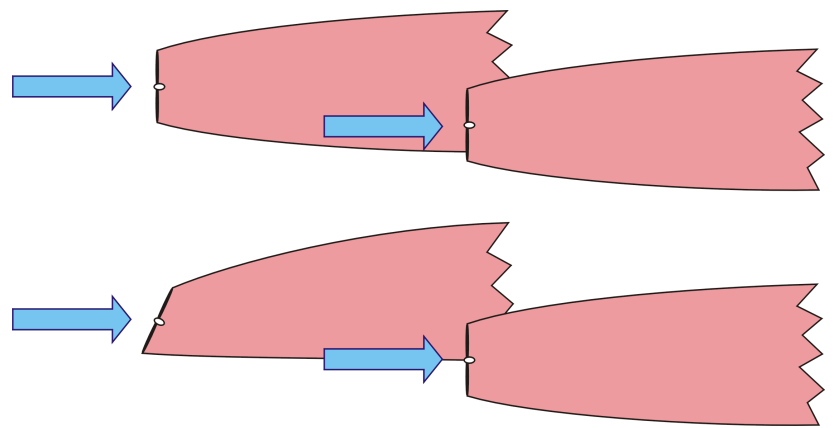

Figure 10. Visualization of the wake redirection introduced by Controlling Wind: normal situation (top), and effect of Controlling Wind on the wake (bottom)

Due to the yaw misalignment introduced, Controlling Wind can increase or decrease the loads on the wind turbines for some wind directions. For this reason, it is important to evaluate the damage equivalent fatigue loads and compare these to those in the reference case without Controlling Wind. To this end, an extensive loads database can be constructed using aeroelastic simulations under many different operating conditions such as a range of wind speeds, turbulence intensities, wake deficit profiles, wake locations, and yaw misalignment.

As of present, Controlling Wind has not yet been fully tested in the field. However, numerous simulation studies indicate its huge potential: ${ }^{29,30}$ even for a row of just 3 turbines, an energy yield increase of more than $10 \%$ can be realized by Controlling Wind when wind turbines are in the half-wake of upstream turbines. For long rows containing many wind turbines, this gain in energy can even reach up to $30 \%$.

In Figure 11 four individual cases of yaw misalignment is presented to show the corresponding wake deviation. In case of $30^{\circ}$ of yaw (bottom right of Figure 11) the wake center deviates around $0.25 D$ from the center line.

In Figure 12 a demonstration is shown for 3 wind turbines. Each downstream turbine is aligned to be at the half-wake of the upstream turbine and the incoming wind direction differs $5^{\circ}$ from the line of turbines. The wake of the last turbine is not simulated since we are only interested in the power production of the wind turbines. The left of Figure 12 shows a line of three turbines in normal operation where each downstream turbine is indeed in the half-wake of the upstream turbine. In the figure on the right the most upwind turbine is yawed $20^{\circ}$ in order to steer its wake away from the downstream turbines. As a result, the wake deficit at the second turbine has been reduced significantly, while also a small benefit for the third turbine is visible. When both figures are compared it can clearly be seen from the figure on the right that the wake of the first turbine is moved away from the centerline so the second turbine is effected less from the half wake situation. This leads to an increase in power and decrease in loads for the second (and hence the other downstream) turbine.

In Figure 13 a comparison of relative power production is shown for the case of 5 wind turbines aligned in a row. For each yaw misalignment case (where only the most upstream turbine is yawed) the total power production of 5 turbines is calculated and compared to the total power production for the case of $0^{\circ}$ yaw angle. It can be seen from this plot that he most effective yaw angle is around $20^{\circ}-22^{\circ}$.

\section{IV.C. Dynamic Active Wake Control}

One of the risks in the application of Active Wake Control (AWC) is the variability of the wind direction in relation to the slow response of the yaw angle of the turbine. This causes uncertainties in the position of the wake with respect to other turbines. In order to gain realistic results from simulations with AWC, it is 

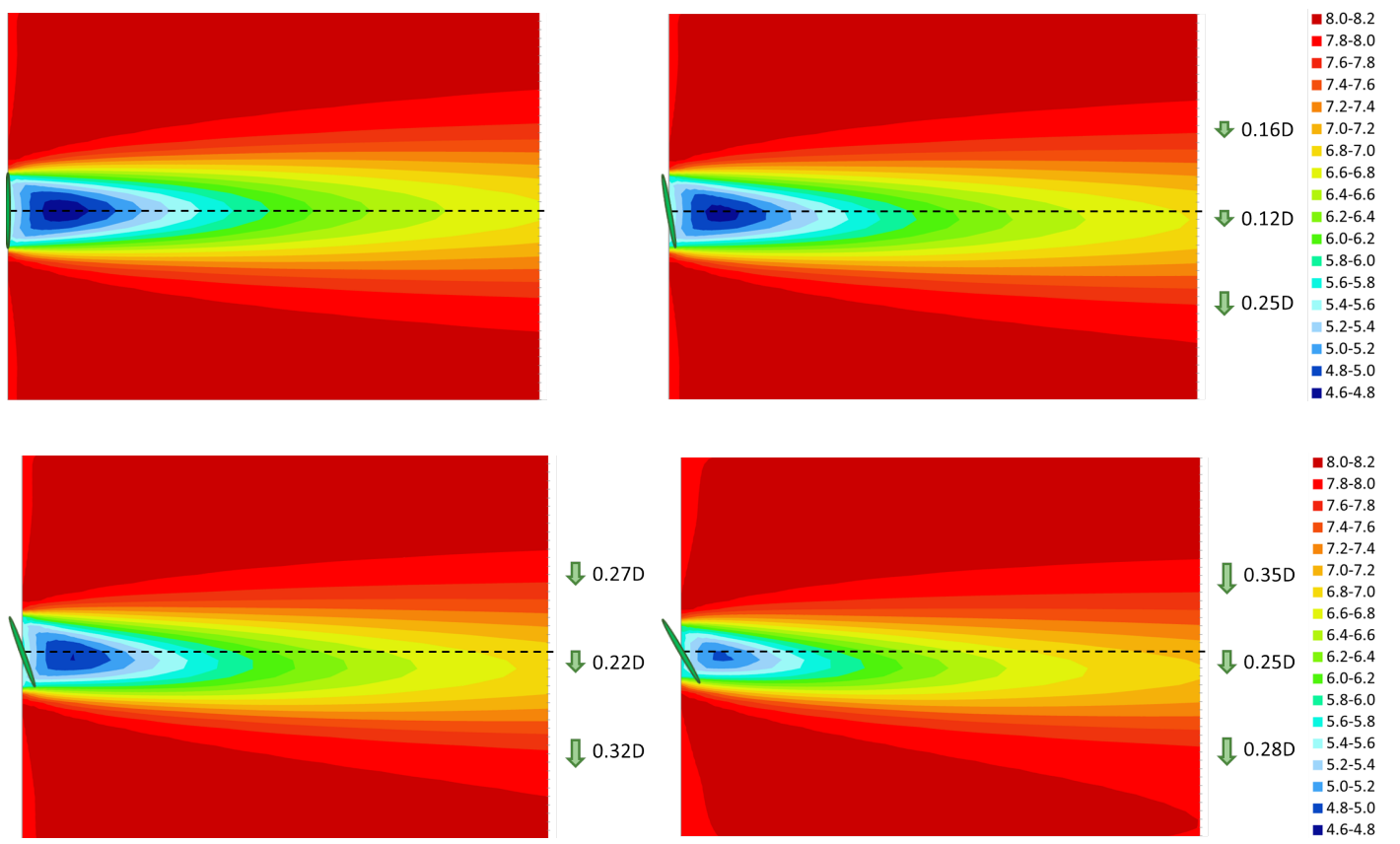

Figure 11. Visualization of the contour plots of velocity indicating the wake deviation for yaw angles of 0 (top left), 10 (top right), 20 (bottom left) and 30 (bottom right) degrees.
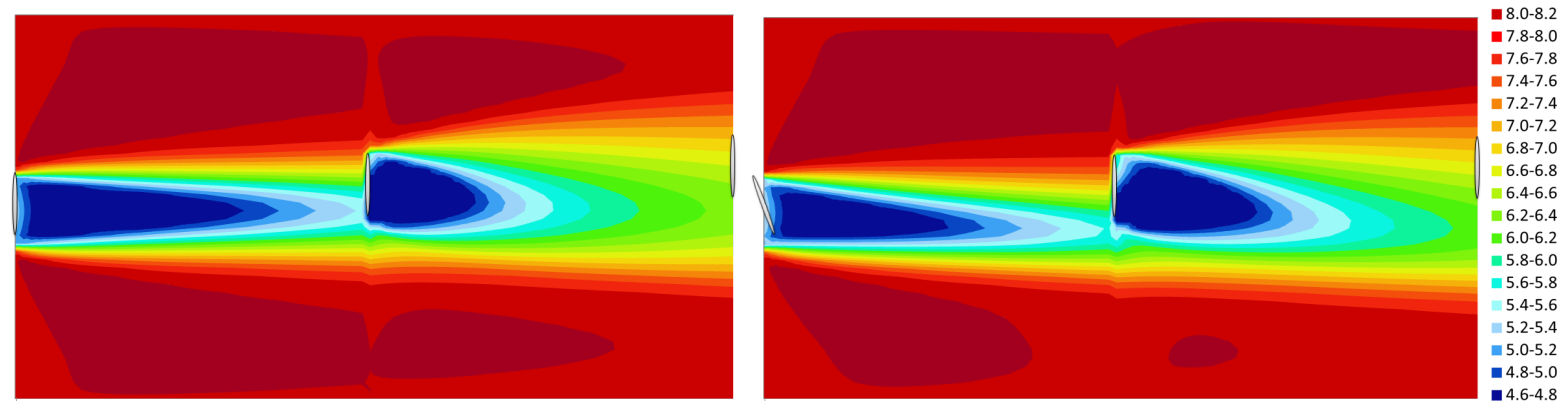

Figure 12. Visualization of the contour plots of velocity in the wake direction where the first turbine in a raw is yawed.

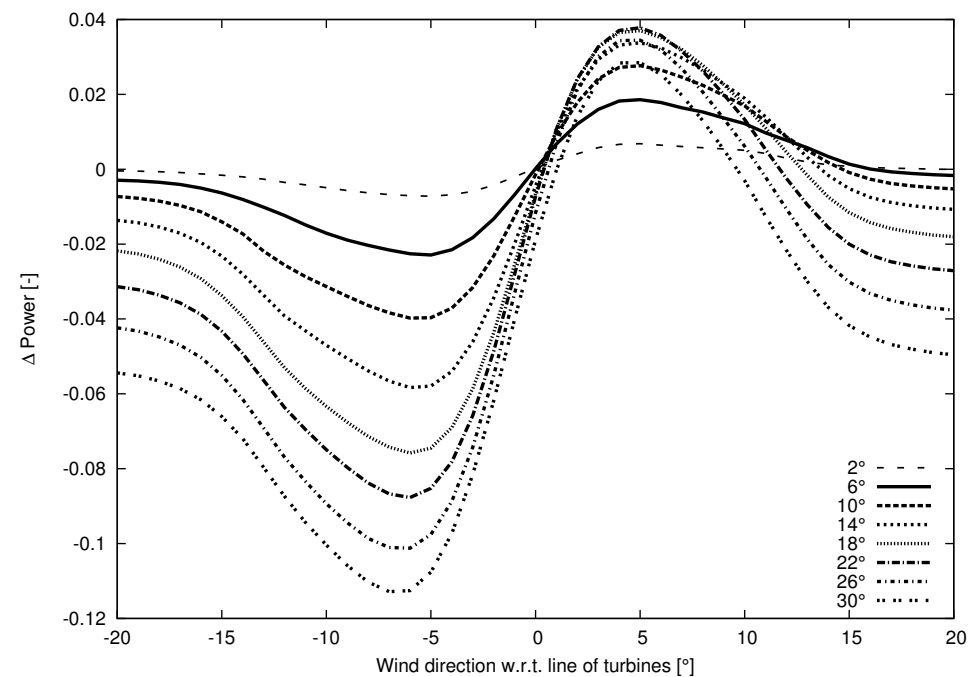

Figure 13. Comparison of relative power production of 5 turbines for various yaw angles with respect to yaw angle of 0 degrees. 
important to account for the uncertainty in the wake trajectory. Therefore, the simulations with AWC are done in a quasi-dynamic way with one-minute time series. The turbines are controlled dynamically on basis of the results of the previous time step. The succeeding time step with a new wind vector, which introduces an unknown change in wind direction and wind speed, is then simulated with these settings. The benefit of AWC is determined by running the simulations with the time series twice: the first time with normal operation and the second time while applying AWC.

\section{Validation case}

FarmFlow has been applied to the same test cases as FlaP in order to compare its results with data derived from the LiDAR measurement campaign in the wind farm Nordsee Ost. Whereas FarmFlow is developed to calculate the (multiple) wake effects throughout large offshore wind farms, no multiple wake test cases could be selected from the measurements due to extreme low availability under multiple wake conditions.

In all FarmFlow calculations, wind speed, wind direction and turbulence intensities are based on the measured values from the met mast. Yaw (misalignment) angles of the turbine are modelled as well in FarmFlow.

Figure (14) shows the measured and the simulated wind field for test case 1. The grid of the FarmFlow results is aligned differently from the LiDAR measurements. The grid of the FarmFlow results is aligned with the wind direction, while the grid of the LiDAR measurements is aligned with the rotor axis. The measured wakes will show a much larger offset from the LiDAR grid, than from the wind direction. The position of the wake will also deflect from the wind direction, however this deflection angle is less than $25 \%$ of the yaw misalignment angle. The wake deficit in the simulation results is lower than the wake deficit that is seen by
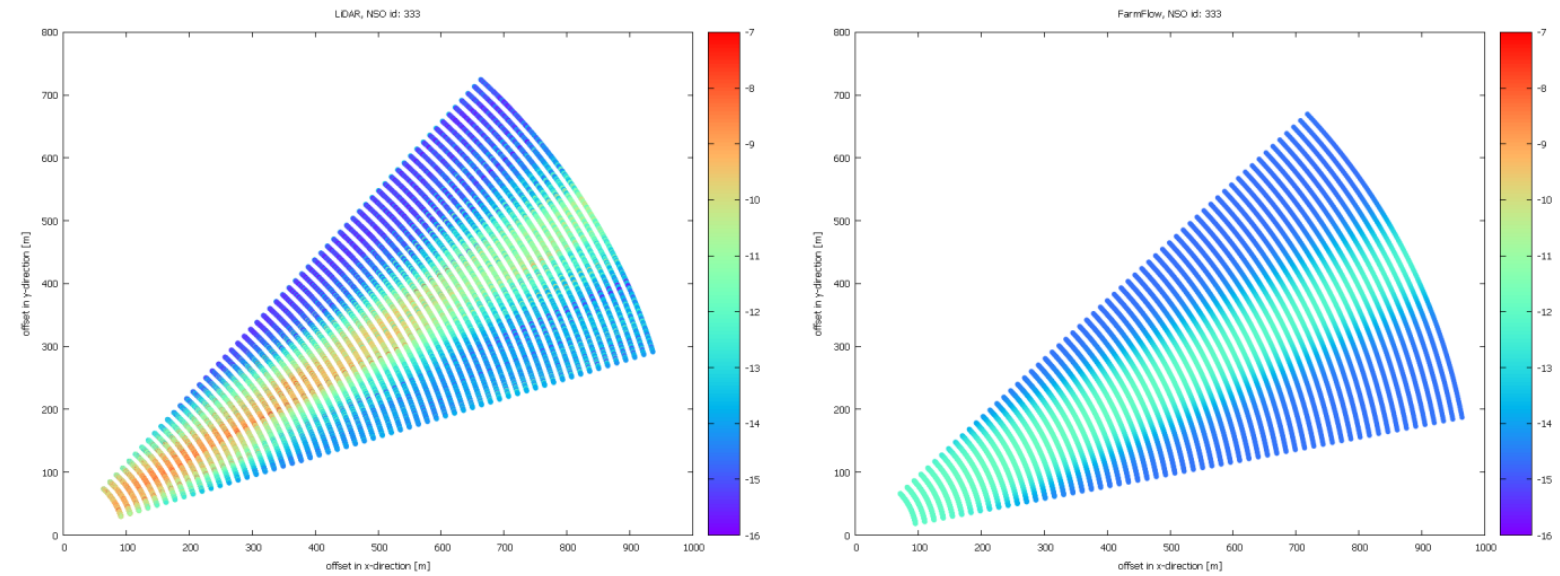

Figure 14. Comparison of wind fields from LIDAR (left) and FarmFlow (right) for test case 1 (NSO-id 333).

the LiDAR, especially at smaller distances behind the rotor and above rated wind speeds. Top-left figure in Figure 15 shows the wind speeds perpendicular to the centre line at a distance of 3D behind the rotor (shown with red solid line). The width of the wake is very well captured by FarmFlow, but at the centre of the wake the velocity deficit is largely underestimated, probably due to underestimated thrust forces near the rotor centre. The average wind speed in the wake centre as measured by the LiDAR is $9.4 \mathrm{~m} / \mathrm{s}$ for case id 333 . With an undisturbed wind speed of $14.59 \mathrm{~m} / \mathrm{s}$, the induction factor according to blade element momentum (BEM) theory the following relationship holds for the far wake (3D behind the rotor can be considered as far wake $)^{31}$

$$
u_{\text {wake }}=(1-2 a) u_{\infty}
$$

where $u_{\text {wake }}$ is the velocity of the wind $3 \mathrm{D}$ behind the rotor, $a$ is the induction factor and $u_{\infty}$ is the undisturbed velocity. Using the given velocity values the induction factor can be calculated as $(1-9.4 / 14.59) / 2=$ 0.178. This corresponds to a theoretical thrust coefficient (ideal wind turbine) of 0.585 where the theoretical thrust coefficient $C_{T}$ is defined by ${ }^{31}$

$$
C_{T}=4 a(1-a)
$$


The actual thrust coefficient according to the official turbine data at $14.59 \mathrm{~m} / \mathrm{s}$ is only 0.357 , which is $39 \%$ lower than the theoretical value of 0.585 . As mentioned above, the most plausible cause for this large discrepancy is an inaccurate thrust coefficient given in the specifications of the wind turbine. The application of a thrust coefficient assumes a uniformly loaded rotor, which is only reasonable (far) below rated wind speeds. Around and above rated wind speeds, when the blades are pitched, the thrust forces are much more reduced near the tip than near the root of the blades.

Similar results at 5D and 7D distance behind the rotor are shown in Figure (15). For FarmFlow it has
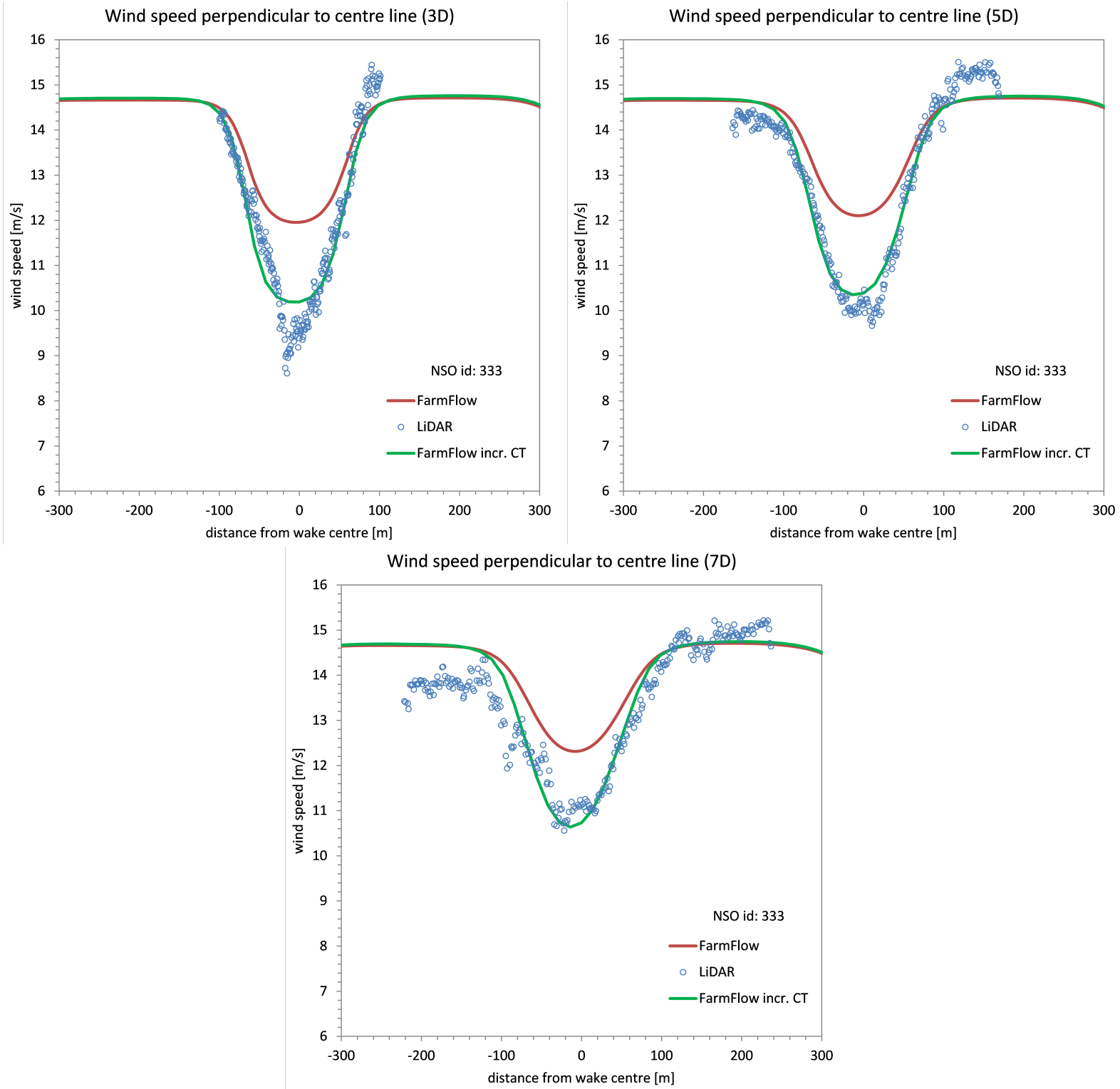

Figure 15. Wind speed at hub height 3D (top-left), 5D (top right) and 7D (bottom) behind the wind turbine along the direction perpendicular to the wind direction provided by the NSO met mast in test case 1 (NSO id 333). The blue circles are LiDAR measurements. The red and green line show the FarmFlow results with original and increased thrust coefficient respectively.

been attempted to improve the agreement between simulated and measured wind fields by increasing the thrust coefficient in the simulations. Instead of using the thrust coefficient specified by the wind turbine manufacturer, an adjusted thrust coefficient has been derived based on the information on the inflow wind speed derived from the met mast and information on the wind speed in the wake derived from the LIDAR measurements, using the relations of blade element momentum theory. For test case 1, NSO id 333 with an undisturbed wind speed of $14.59 \mathrm{~m} / \mathrm{s}$, the simulations were carried out with an adjusted thrust coefficient of 0.45 instead of the the thrust coefficient given by the wind turbine specifications of 0.357 . 
Top-left figure in Figure (15) shows the profiles of the wind speeds perpendicular to the centre line at a distance of 3D downstream (shown with green solidline). It is obvious that the comparison of the FarmFlow simulations with LiDAR measurements is largely improved when the applied thrust coefficient is derived from BEM theory taking into account the wake velocities in the near wake region, and calculating the induction factor. Also the comparisons for distances $5 D$ and $7 D$ have improved considerably (top-rigth and bottom figures). Both width of the wake and wake velocity at the centre line are very accurate. Only at a distance of $3 D$ the measured wake is smaller and asymmetrical near the centre line with a lower minimum velocity. Nevertheless, the average value of the curve near the centreline shows good agreement with the simulations.

The asymmetry at the edge of the wake, most clearly seen at $5 D$ and $7 D$ downstream, is possibly an effect from wake rotation in combination with vertical shear. Air with the higher velocity at the top of the wake due to the vertical shear shifts towards the right side of the wake due to the wake rotation. At the same time, the lower velocity at the bottom of the wake shifts towards the left side of the wake.

\section{Conclusions}

In this study improvements to ECN wake model, WakeFarm, are presented in three folds. First of all, an improved near wake model is shown where the wake is modeled by a thin vortex sheet represented by discrete vortex rings of variable strength. The solution is obtained analytically with the Biot-Savart law, where the elliptic integrals are evaluated numerically. It is shown that the induced velocity is higher than for the original near wake model and more in accordance with the theory. Furthermore the diabatic wind profile model is improved by implementing a model valid for the entire boundary layer as suggested by Gryning et al. ${ }^{16}$ The results are compared with a diabatic wind model valid for the surface layer and with the data obtained from EWTW measurements. Although the results seem closer to the data, the EWTW data is a single data set representing all stability regions. An initial attempt to categorize the EWTW data into two stability regions depending on the time of the day of the measured data did not lead to better conclusions. A need for a better data mining to distinguish the different stability regions and data from different sites is obvious. In addition, accurate temperature measurements should be standard for wake measurement campaigns. Finally the concept of Active Wake Control (AWC) is explained and it is shown that AWC can be simulated in a quasi-dynamic way using wind time series with a 1-minute time step in order to account for uncertainties in the variable wind conditions. A comparison of the model with the wind field data obtained by Lidar measurements is shown and the discrepancy between the theoretical and actual thrust coefficients of the wind turbine is discussed.

\section{Acknowledgements}

This project has received funding from the European Unions Horizon 2020 research and innovation program under grant agreement No 727477 (project CL-Windcon).

\section{References}

\footnotetext{
${ }^{1}$ Schepers, J. G., "Wakefarm: nabij zog model en ongestoord wind snelheidsveld," Tech. Rep. ECN-C-98-016, ECN, 1998.

${ }^{2}$ van der Pijl, S. P. and Schepers, J. G., "Improvements of the WAKEFARM Wake Model," Workshop on wake modelling and benchmarking of models, Annex XXIII: Offshore wind energy technology and deployment, Billund, 2006.

${ }^{3}$ Schepers, J. G. and van der Pijl, S. P., "Improved modelling of wake aerodynamics and assessment of new farm control strategies," The Science of Making Torque from Wind, Journal of Physics: Conference series 75, 2007.

${ }^{4}$ Özdemir, H., Versteeg, M. C., and Brand, A. J., "Improvements in ECN Wake Model," ICOWES2013, Lyngby, Denmark, 17-19 June 2013.

${ }^{5}$ Crespo, A., Manuel, F., Moreno, D., Fraga, F., and Hernáandez, J., "Numerical analysis of wind turbine wakes," Delphi Workshop on Wind turbine applications, 1985.

${ }^{6}$ Crespo, A. and Hernández, J., "Numerical modelling of the flow field in a wind turbine wake," 3rd Joint ASCE/ASME Mechanics Conference, 1989.

${ }^{7}$ Monin, A. S. and Obukhov, A. M., "Basic laws of turbulent mixing in the surface layer of the atmosphere," Tr. Akad. Nauk SSSR Geofiz. Inst., Vol. 24, 1954, pp. 163-187, English translation by John Miller, 1959.

${ }^{8}$ Businger, J. A., Wjngaard, J. C., Izumi, Y., and Bradley, E. F., "Flux-profile relationships in the atmospheric surface layer," Journal of the Atmospheric Sciences, Vol. 28, 1971, pp. 181-189.
} 
${ }^{9} \varnothing$ ye, S., "A simple vortex model," 3rd IEA Symposium on the Aerodynamics of Wind Turbines, pp. 4.1-4.15.

${ }^{10}$ Grachev, A. A., Fairall, C. W., and Bradley, E. F., "Convective profile constants revisited," Boundary-Layer Meteorology, Vol. 94, No. 3, 2000, pp. 495-515.

${ }^{11}$ Seibert, P., Beyrich, F., Gryning, S. E., Joffre, S., Rasmussen, A., and Tercier, P., "Review and intercomparison of operational methods for the determination of the mixing height," Atmospheric Environment, Vol. 34, No. 7, 2000 , pp. 1001 1027.

${ }^{12}$ Gárcia, M. A., Sánchez, M. L., de Torre, B., and Pérez, I. A., "Characterisation of the mixing height temporal evolution by means of a laser dial system in an urban area intercomparison results with a model application," Ann. Geophys., Vol. 25, 2007, pp. 21192124.

${ }^{13}$ Emeisa, S., Münkelb, C., Vogtc, S., Müllerd, W. J., and Schäfera, K., "Atmospheric boundary-layer structure from simultaneous SODAR, RASS, and ceilometer measurements," Atmospheric Environment, Vol. 38, No. 2, 2004, pp. 273-286.

${ }^{14}$ Blackadar, A. K., "The vertical distribution of wind and turbulent exchange in a neutral atmosphere," Journal of Geophysical Research, Vol. 67, No. 8, 1962, pp. 3095-3102.

${ }^{15}$ Lettau, H. H., "Studies of the three-dimensional structure of the planetary boundary layer," Tech. Rep. AD-296160, University of Wisconsin, Department of Meteorology, 1962.

${ }^{16}$ Gryning, S. E., Batchvarova, E., Brümmer, B., Jørgensen, H., and Larsen, S., "On the extension of the wind profile over homogeneous terrain beyond the surface boundary layer," Boundary-Layer Meteorology, Vol. 124, No. 2, 2007, pp. 251-268.

${ }^{17}$ Peña, A., Gryning, S. E., and Hasager, C. B., "Comparing mixing-length models of the diabatic wind profile over homogeneous terrain," Theoretical and Applied Climatology, 2010.

${ }^{18}$ Sathe, A., Mann, J., Barlas, T., Bierbooms, W. A. A. M., and van Bussel, G. J. W., "Influence of atmospheric stability on wind turbine loads," Wind Energy Journal, Vol. DOI:10.1002/we.1528, 2012.

${ }^{19}$ Patankar, S. V. and Spalding, D. B., "A calculation procedure for heat, mass and momentum transfer in three-dimensional parabolic flows," Int. J. Heat Mass Transfer, 1972.

${ }^{20}$ Machielse, L. A. H., Barth, S., Bot, E. T. G., Hendriks, H. B., and Schepers, G. J., "Evaluation of Heat \& Flux farm control," Tech. Rep. ECN-E-07-105, ECN, 2007.

${ }^{21}$ Panofsky, H. and Dutton, J., Atmospheric Turbulence, John Wiley and Sons, 1984.

${ }^{22}$ Garratt, J. R., The atmospheric boundary layer, Cambridge, 1992.

${ }^{23}$ Peña, A., Gryning, S. E., and Hasager, C. B., "Measurement and modelling of the wind speed profile in the marine atmospheric boundary layer," Boundary-Layer Meteorology, Vol. 129, 2008, pp. 479-495.

${ }^{24}$ Peña, A., Gryning, S. E., Mann, J., and Hasager, C. B., "Length scales of the neutral wind profile over homogeneous terrain," Journal of Applied Meteorology and Climatology, Vol. 49, 2010, pp. 792-806.

${ }^{25}$ Corten, G. P. and Schaak, P., "Method and installation for extracting energy from a flowing fluid, patent number WO2004/011799," Tech. rep., 2004.

${ }^{26}$ Küçükşahin, D., "Heat and Flux configuration on offshore wind farms," Tech. Rep. ECN-X-12-079, ECN, Petten, The Netherlands, 2012.

${ }^{27}$ Corten, G. P. and Schaak, P., "Heat and Flux; Increase of wind farm production by reduction of the axial induction," Proceedings of the European Wind Energy Conference, Madrid, Spain, 2003.

${ }^{28}$ Corten, G. P., Lindenburg, K., and Schaak, P., "Assembly of energy ow collectors, such as windpark, and method of operation, patent number WO2004/011799," Tech. rep., 2002.

${ }^{29}$ Gebraad, P. M. O., Teeuwisse, F. W., Wingerden, v. J. W., Fleming, P. A., Ruben, S. D., Marden, J. R., and Pao, L. Y., "A Data-Driven Model for Wind Plant Power Optimization by Yaw Control," Proceedings of the American Control Conference, 2014.

${ }^{30}$ Fleming, P., Gebraad, P., Lee, S., Wingerden, v. J. W., Johnson, K., Churchfield, M., Michalakes, J., Spalart, P., and Moriarty, P., "High-fidelity simulation comparion of wake mitigation control strategies for a two-turbine case," ICOWES2013 Conference, Lyngby, 17-19 June 2013.

${ }^{31}$ Hansen, M. O. L., Aerodynamics of Wind Turbines, Earthscan, 2008. 